\title{
Optimizing the Packing Density and Chemistry of Cellulose Nanofilters for High-Efficiency Particulate Removal
}

Shao-Hsiang Hung ${ }^{1}$, Jared W. Bowden ${ }^{1}$, Richard E. Peltier ${ }^{2}$, and Jessica D. Schiffman ${ }^{1 *}$

1Department of Chemical Engineering, University of Massachusetts Amherst, Amherst, Massachusetts 01003-9303, United States

${ }^{2}$ School of Public Health \& Health Sciences, University of Massachusetts Amherst, Amherst, Massachusetts 01003-9303, United States

${ }^{*}$ Corresponding author: Jessica D. Schiffman

Email: schiffman@ecs.umass.edu; Phone: (413) 545-6143 
Table S1. Summary of the elemental data analysis of the high resolution XPS that provides surface chemical composition analysis of cellulose, PDA, and PDADMAC electrospun nanofilters.

\begin{tabular}{|c|c|c|c|}
\hline Nanofilter & $\mathbf{C ~ ( \% ) ~}$ & $\mathbf{O}(\%)$ & $\mathbf{N}(\%)$ \\
\hline Cellulose & 59.1 & 38.6 & 0.1 \\
\hline PDA & 64.0 & 33.4 & 2.5 \\
\hline PDADMAC & 65.8 & 31.5 & 1.5 \\
\hline
\end{tabular}



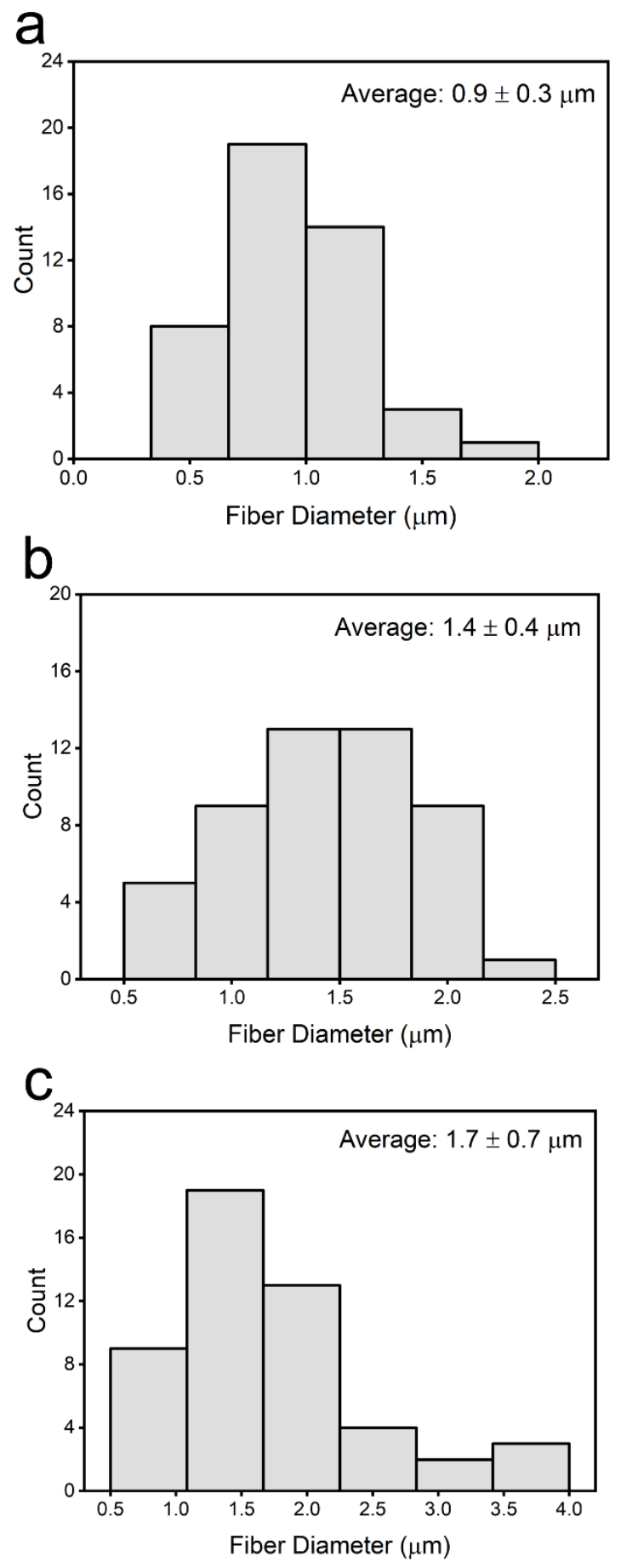

Figure S1. Fiber diameter distribution and average fiber diameter of (a) cellulose (b) PDAfunctionalized, and (c) PDADMAC-functionalized nanofilters. 

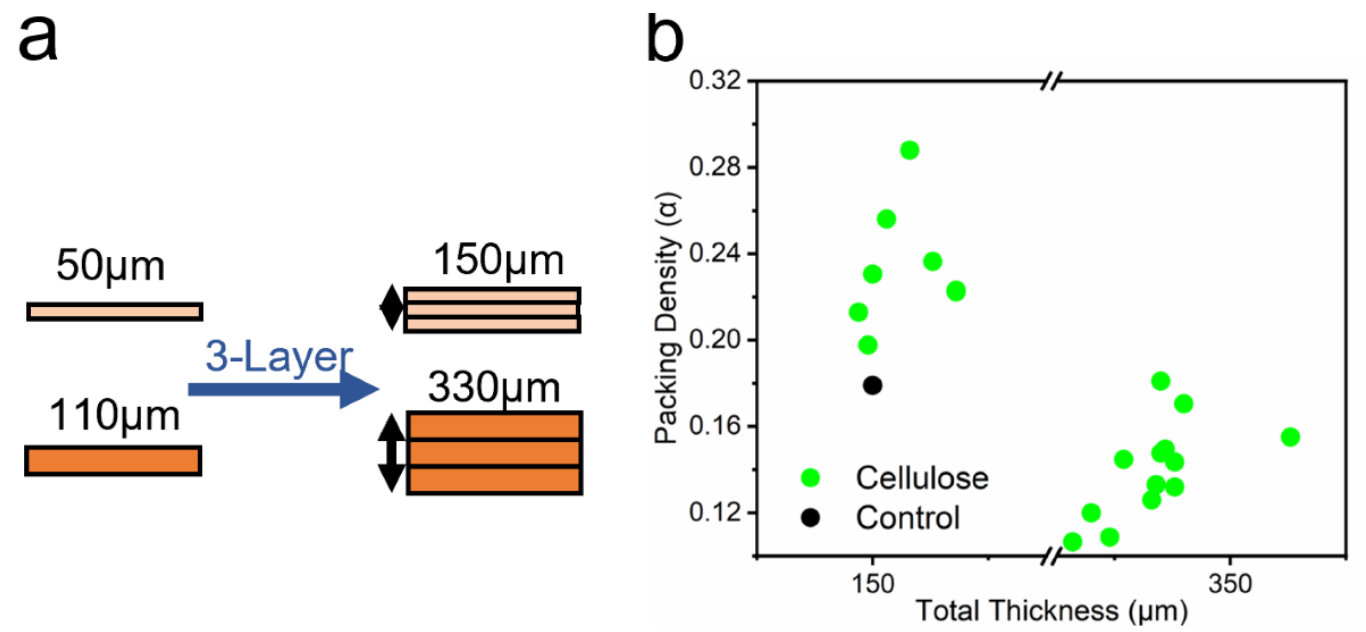

Figure S2. (a) Schematic diagram of single and multilayer electrospun nanofilters with different thicknesses. (b) Packing density as a function of total thickness for MCE controls and all cellulose nanofilters investigated in this work.
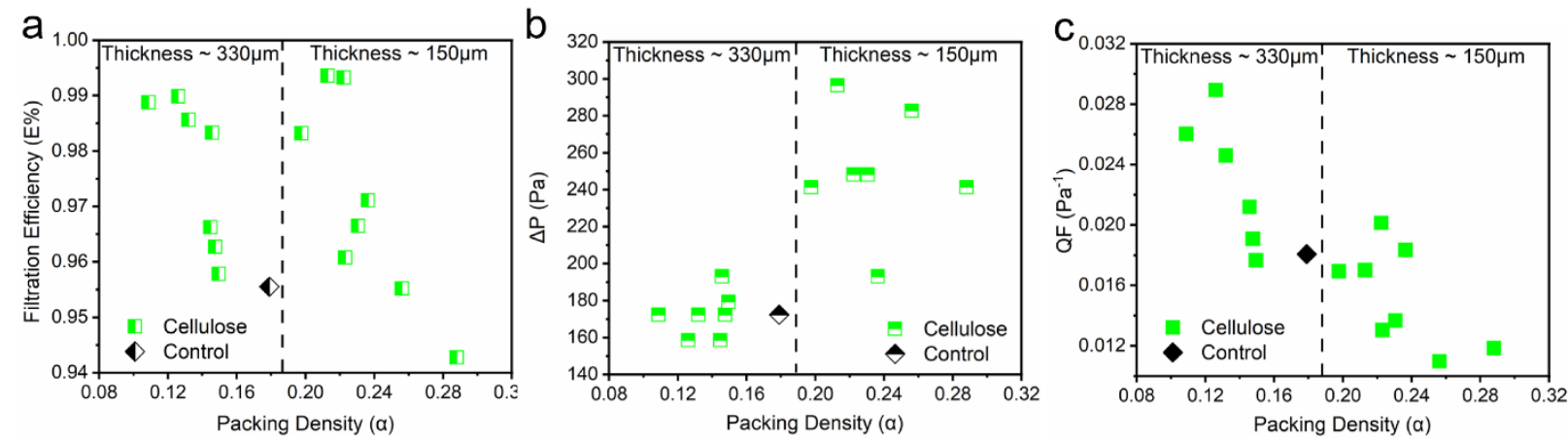

Figure S3. (a) Filtration efficiency (b) Pressure drop and (c) Quality factor as a function of packing density for the MCE control, and cellulose nanofilters. The dotted line separates the filters that had a total bulk thickness of $330 \mu \mathrm{m}$ from those that were 150 $\mu \mathrm{m}$ thick.
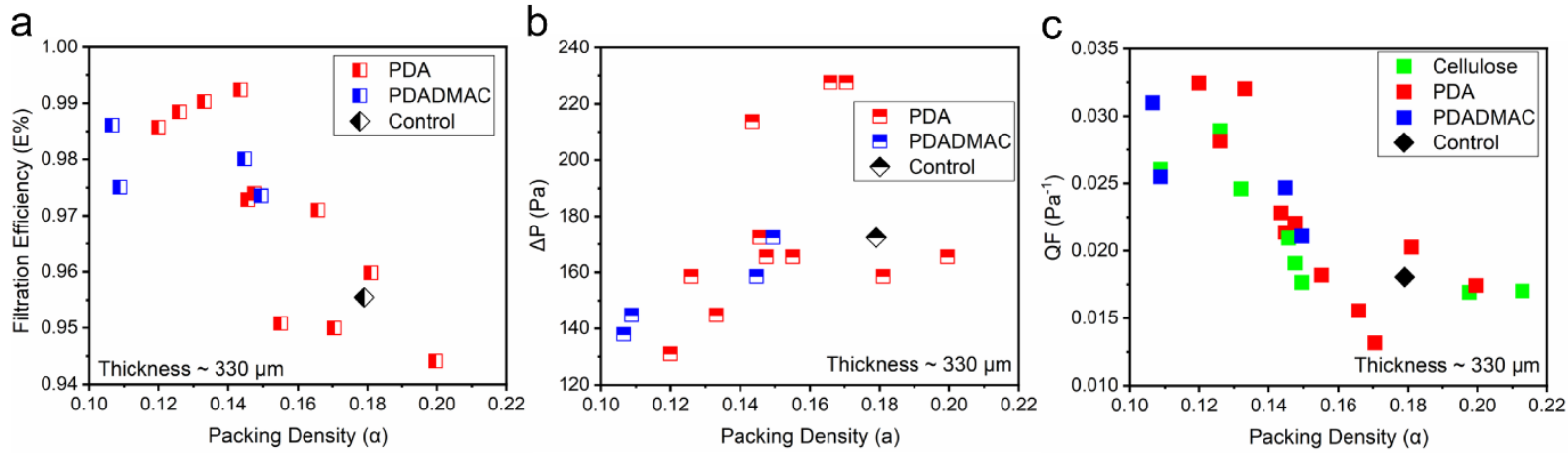

Figure S4. (a) Filtration efficiency (b) Pressure drop and (c) Quality factor as a function of packing density for the MCE control, PDA and PDADMAC samples. All samples had a total filter thickness of $330 \mu \mathrm{m}$. 